\title{
Comparação de estratégias para aprimoramento de graduandos de Odontologia na prescrição medicamentosa
}

\author{
Tamires Baptista Scarpin*; Luciana Butini Oliveira**; Cristiane de Cássia Bergamaschi***; Juliana \\ Cama Ramacciato****; Rogério Heládio Lopes Motta****
}

* Graduada em Odontologia, Faculdade São Leopoldo Mandic

** Docente, Área de Odontopediatria, Faculdade São Leopoldo Mandic

*** Docente, Área de Ciências Farmacêuticas, Universidade de Sorocaba

**** Docente, Área de Farmacologia, Anestesiologia e Terapêutica, Faculdade São Leopoldo Mandic

Recebido em 10/04/2018. Aprovado em 23/02/2019.

\begin{abstract}
RESUMO
O objetivo deste estudo foi avaliar diferentes estratégias para o aprimoramento de graduandos de Odontologia sobre normas de prescrição medicamentosa. Foram avaliados 72 estudantes, os quais foram divididos aleatoriamente em 3 grupos $(n=24)$ : Grupo 1 - em cada etapa de avaliação foi apresentado um vídeo explicativo sobre normas de prescrição com duração de 15 minutos; Grupo 2 - o mesmo vídeo foi disponibilizado em uma plataforma virtual (com acesso ilimitado); Grupo 3 controle: os estudantes não tiveram atividades adicionais sobre o tema. Foram realizadas 4 avaliações e os voluntários receberam um caso clínico diferente em cada avaliação, realizando uma prescrição para a situação clínica proposta. Para cada prescrição realizada foi atribuída uma pontuação considerando diferentes itens: identificação do profissional e do paciente, concentração, dose e quantidade do medicamento, instruções, dentre outros. Os resultados obtidos foram submetidos à análise estatística, com nível de significância de 5\%. Não foram observadas diferenças significativas na avaliação basal entre os grupos ( $p>0,05)$. Após o uso das diferentes estratégias foram observados melhores resultados para G1 quando comparados a G2 e G3 (p<0,05). Concluiuse que o uso do vídeo presencial foi o que proporcionou melhores resultados em relação às normas de prescrição de medicamentos.
\end{abstract}

Descritores: Educação em Odontologia. Prescrições de Medicamentos. Odontologia. Ensino. 


\section{INTRODUÇÃO}

Segundo a Organização Mundial de Saúde (OMS) muitos medicamentos são prescritos ou comercializados de forma inapropriada, sendo necessárias medidas para conter este problema ${ }^{1}$. Além do impacto deletério sobre a saúde dos pacientes, o uso inadequado de medicamentos agrava o acesso da população a esses agentes terapêuticos, os quais, se bem indicados e utilizados, ajudam a reduzir a morbidade e mortalidade dos usuários do sistema de saúde, indistintamente, na esfera pública ou privada. Neste contexto, a prescrição de medicamentos é de extrema relevância para o seu uso racional e seguro pela população ${ }^{2}$.

A "receita médica" é um documento escrito que retrata a conclusão de um raciocínio clínico a partir de dados coletados por meio de entrevista criteriosa, bem como a avaliação física, contribuindo para o sucesso do resultado clínico e do tratamento oferecido ${ }^{3}$.

A "prescrição de medicamentos" é definida como uma ordem escrita dirigida ao farmacêutico, que esclarece como $\mathrm{o}$ fármaco deve ser fornecido ao paciente, determinando as condições de seu uso ${ }^{4}$. Deve sempre ser apresentada por escrito, já que responsabiliza tanto quem prescreve quanto quem dispensa, e deve ser legível para que haja entendimento correto do conteúdo prescrito, evitando má interpretação ${ }^{5}$.

Além da clareza para o paciente, a prescrição medicamentosa também deve seguir os critérios da OMS para prescrição racional, sendo apropriada, segura, efetiva e econômica ${ }^{6}$. De acordo com o decreto 20.931 de 11 de janeiro de 1932, a prescrição de medicamentos é um dos deveres do profissional médico, do cirurgião-dentista e do médico veterinário. Considerando o número de prescritores com diferentes qualificações na área da saúde, torna-se cada vez mais importante sua capacitação para escolher e prescrever medicamentos de forma racional ${ }^{7}$.
Poucos estudos avaliam a prescrição de medicamentos na prática clínica da Odontologia. Um dos motivos para este cenário talvez seja devido ao consenso de que o cirurgião-dentista prescreve medicamentos por um período mais curto e que o seu arsenal terapêutico é restrito ${ }^{8}$. A Lei 5.081 de 1966 que regulamenta o exercício da Odontologia no Brasil determina, no art. 6, item II: "Compete ao cirurgião-dentista prescrever e aplicar especialidades farmacêuticas de uso interno e externo, indicadas em Odontologia". Também é dever legal do cirurgião-dentista conhecer as características farmacológicas dos medicamentos que prescreve, devendo sempre analisar criteriosamente a informação oferecida pelos laboratórios farmacêuticos, bem como os possíveis efeitos apresentados pelo uso destes medicamentos $^{9,10}$.

O fato é que a prescrição de medicamentos ainda gera muitas dúvidas para estudantes de Odontologia em relação à quando e como utilizálos, sendo de fundamental importância conhecer a maneira com estes futuros profissionais elaboram suas prescrições ${ }^{11,12}$.

Considerando este panorama atual sobre o conhecimento de estudantes de Odontologia quanto à prescrição medicamentosa, se faz necessária a busca por métodos que possam contribuir para a sua capacitação ${ }^{13,14}$. Desta forma, este estudo teve como objetivo avaliar diferentes estratégias de ensino voltadas para o aprimoramento do conhecimento de estudantes de Odontologia em relação às normas de prescrição de medicamentos.

\section{METODOLOGIA}

O estudo foi conduzido de acordo com os preceitos determinados pela resolução 466/12 do Conselho Nacional de Saúde do Ministério da Saúde e pelo Código de Ética Profissional Odontológico, segundo a resolução CFO 179/93, tendo sido aprovado pelo Comitê de Ética de 
Pesquisa da Faculdade São Leopoldo Mandic, CAAE 46717415.0.0000.5374.

Cada voluntário recebeu um termo de consentimento livre e esclarecido contendo os propósitos da pesquisa, bem como a garantia do sigilo sobre as informações ali prestadas, a garantia do anonimato e do uso exclusivo dos dados para fins de pesquisa. Somente após a sua anuência, por meio da assinatura do termo, o estudante foi considerado participante da pesquisa.

Foram convidados a participar do estudo 90 estudantes regularmente matriculados na Faculdade São Leopoldo Mandic. Foram incluídos no presente estudo aqueles que concordaram em participar da pesquisa e que já tivessem cursado a disciplina de Terapêutica Medicamentosa I, ministrada no $4^{\circ}$ período letivo. Desta forma, participaram os estudantes do $4^{\circ}, 6^{\circ}$ e $8^{\circ}$ períodos.

Somente foram considerados os voluntários que participaram de todos os estágios de treinamento e avaliação. Assim, 72 estudantes concluíram todas as etapas do estudo.

O sorteio dos grupos foi realizado antes da aplicação do teste basal, a fim de não tornar a divisão tendenciosa em relação aos resultados obtidos. Os alunos foram divididos em três grupos por período, e cada grupo recebeu uma metodologia de ensino diferente: Grupo $1(n=24)$ foi exibido, a cada 2 meses, um vídeo explicativo com duração de 15 minutos, mostrando o passo a passo com informações essenciais para a correta prescrição de medicamentos; Grupo $2(n=24)$ - o mesmo vídeo foi disponibilizado em uma plataforma virtual na Internet com acesso ilimitado; e Grupo 3 (Controle, $n=24$ ) - os participantes somente foram submetidos às mesmas avaliações e com a mesma frequência, não tendo recebido conteúdos adicionais ao ministrado regularmente no Curso de Graduação.

No teste basal realizou-se a primeira avaliação de prescrição para um caso clínico hipotético, no qual foi aferida a performance inicial de cada participante, independentemente do grupo. As demais avaliações também foram por meio de casos clínicos interpretados pelos estudantes, que no final da atividade realizaram uma prescrição para o caso proposto. A cada avaliação foi utilizado um caso clínico diferente, a fim de evitar que os estudantes aumentassem a taxa de acerto devido à memorização das questões. O grau de dificuldade e as características da informação foram semelhante em todos os testes, os quais foram realizados nos horários livres dos estudantes.

Cada avaliação foi realizada ao final do bimestre, antes da atividade didática correspondente, visando reduzir possível viés no estudo. No total, foram realizadas 4 avaliações: uma basal e outras 3 para testar a eficácia das diferentes estratégias de ensino.

Para cada prescrição foi atribuída uma pontuação máxima de 5 pontos $^{15}$ (quadro 1 ). Os resultados obtidos foram submetidos aos testes de Wilcoxon e Kruskall-Wallis, com nível de significância de $5 \%$.

\section{RESULTADOS E DISCUSSÃO}

Como a hipótese inicial do estudo era avaliar se alguma das estratégias propostas poderia aprimorar o conhecimento dos estudantes em relação às normas de prescrição, comparou-se a pontuação das fases inicial e final do estudo (tabela $1)$.

Nos grupos 2 e 3 foi possível observar piora na pontuação em relação à avaliação basal, demonstrando portanto que o grupo 1 (vídeo presencial) foi o que mostrou o melhor resultado.

Este resultado é similar ao observado por outros autores ${ }^{16}$, que demonstraram que a implementação de vídeos melhorou o desempenho de estudantes de medicina na prescrição de medicamentos. Dentro deste contexto, o vídeo presencial serviu como um reforço do conteúdo direcionado para as normas de prescrição, uma vez que independentemente do 
caso clínico proposto os estudantes deste grupo apresentaram uma sensível melhora em relação à pontuação obtida na fase basal do estudo. Além disso, os resultados do presente estudo corroboram com outros pesquisadores ${ }^{17}$, que observaram que aulas expositivas adicionais sobre o tema proporcionaram melhora na qualidade das prescrições em relação ao grupo controle dos alunos que tiveram apenas o conteúdo programático curricular.

Quadro 1. Itens de avaliação e respectivas pontuações

\begin{tabular}{|c|c|c|}
\hline $\begin{array}{r}\text { do profissional } \\
\text { do paciente }\end{array}$ & $\begin{array}{l}(0,5 \text { ponto }) \\
(0,5 \text { ponto })\end{array}$ & 1 ponto \\
\hline $\begin{array}{l}\text { Direção farmacêutica } \\
\qquad \begin{array}{r}\text { forma farmacêutica } \\
\text { concentração } \\
\text { quantidade }\end{array}\end{array}$ & $\begin{array}{l}(0,5 \text { ponto }) \\
(0,5 \text { ponto }) \\
(0,5 \text { ponto })\end{array}$ & 1,5 pontos \\
\hline $\begin{array}{r}\text { Informações ao usuário } \\
\text { modo de administração } \\
\text { posologia } \\
\text { duração do tratamento } \\
\text { avisos } \\
\text { data da prescrição e período de uso }\end{array}$ & $\begin{array}{l}(0,5 \text { ponto }) \\
(0,5 \text { ponto }) \\
(0,5 \text { ponto }) \\
(0,5 \text { ponto }) \\
(0,5 \text { ponto })\end{array}$ & 2,5 pontos \\
\hline
\end{tabular}

Tabela 1. Média e desvio padrão dos resultados obtidos nas fases inicial e final do estudo

\begin{tabular}{lccc}
\hline \multicolumn{1}{c}{ Grupos } & Pontuação Inicial & Pontuação Final & Valor de p \\
\hline 1 (Vídeo presencial) & $3,56 \pm 0,75 \mathrm{Aa}$ & $3,98 \pm 0,82 \mathrm{Aa}$ & $\mathbf{0 , 8 1 3 7}$ \\
2 (Vídeo na plataforma) & $3,62 \pm 0,64 \mathrm{Aa}$ & $3,02 \pm 1,47 \mathrm{Bb}$ & $\mathbf{0 , 0 3 2 1}$ \\
3 (Controle) & $3,64 \pm 0,59 \mathrm{Aa}$ & $3,14 \pm 1,15 \mathrm{Bb}$ & $\mathbf{0 , 0 3 7 3}$ \\
\hline Valor de $\mathbf{p}$ & $\mathbf{0 , 9 5 3 9}$ & $<\mathbf{0 , 0 5}$ & \\
\hline
\end{tabular}

Letras maiúsculas diferentes representam diferença estatística nas linhas (Inicial x Final - Teste de Wilcoxon, p <0,05). Letras minúsculas diferentes representam diferença estatística nas colunas (entre os grupos - Teste de Kruskal-Wallis, p <0,05).

Embora outros autores $^{18,19}$ tenham resultado, sendo que os estudantes deste grupo observado que o uso de recursos e plataformas tiveram uma performance similar aos do grupo virtuais podem proporcionar uma melhora na controle. Além disso, é importante ressaltar que aprendizagem de graduandos em Odontologia, após as avaliações aqueles que tinham acesso à no presente estudo o uso da plataforma virtual plataforma recebiam uma mensagem de de livre acesso não proporcionou o mesmo lembrete para o seu uso, e mesmo assim foi 
observado que a maioria dos voluntários pouco acessou a plataforma.

Uma possível justificativa para estes resultados pode ser o fato de que o material explicativo tinha o mesmo conteúdo informativo ao longo do estudo, enquanto qu e no estudo de Gonzales e Gadbury-Amyot ${ }^{19}$, novos materiais e imagens eram adicionados para despertar o interesse. Desta forma, o presente estudo demonstra que o fato de disponibilizar conteúdo em plataformas virtuais não garante sua ampla utilização pelos estudantes, e demonstra a necessidade de ajustes nesta estratégia de ensino para o tema proposto. Adicionalmente, estudo recente ${ }^{20}$ apontou que embora o uso da tecnologia digital já seja uma realidade no Brasil, a sua utilização para fins de estudo ainda parece não ser utilizada por grande parte da população.

As avaliações do presente estudo mostram melhora significativa na qualidade das prescrições realizadas pelo grupo que assistiu presencialmente à vídeo aula, o que corrobora com estudos prévios sobre a necessidade de novas abordagens teóricas e práticas durante a graduação $^{11,12}$, além de mudanças na metodologia de ensino para que os futuros profissionais sejam conscientes sobre seu papel na sociedade e o uso correto de medicamentos. Além disso, uma revisão sistemática $^{21}$ demonstrou que embora algumas estratégias de ensino tenham levado a melhoras na prescrição, ainda são necessários mais estudos que possam auxiliar no aprimoramento das prescrições realizadas por profissionais da área de saúde.

\section{CONCLUSÃO}

Concluiu-se que, dentre as estratégias utilizadas, o uso do vídeo presencial foi o que proporcionou o aprimoramento dos estudantes em relação às normas de prescrição de medicamentos.

\section{AGRADECIMENTO}

Ao Programa Institucional de Bolsas de Iniciação Científica da Faculdade São Leopoldo Mandic pelo apoio para a realização deste estudo (\#PIC 014-2015).

\section{ABSTRACT \\ Comparison of different teaching strategies to improve the knowledge of Dental students about prescribing standards}

The aim of this study was to evaluate different teaching strategies to improve the knowledge of dental students about prescribing standards. We evaluated 72 students who were randomly separated into 3 groups $(n=24)$ : Group 1 - at each evaluation stage, an explanatory video about prescription rules was presented; Group 2 - the same video was made available on a virtual platform (with unlimited access); Group 3 control: the students did not have additional activities on the subject. During the study period 4 evaluations were performed, and all participants received a different clinical case to carry out a prescription for the proposed clinical situation. For each prescription performed, a score was given to different items: identification of professional and patient, concentration, dosage and quantity of the medicine, instructions, among others. The results were submitted to statistical analysis and the level of significance was set at $5 \%$. No significant differences were observed at baseline between groups ( $p>0.05)$. However, after using the strategies better results were observed for G1 when compared with G2 and G3 $(\mathrm{p}<0.05)$. It could be concluded that among the strategies used, the use of video was the one that provided an improvement in knowledge of dental students in relation to prescribing standards.

Descriptors: Dental education. Prescription. Dentistry. Teaching.

\section{REFERÊNCIAS}

1. Donaldson LJ, Kelley ET, Dhingra-Kumar 
N, Kieny MP, Sheikh A. Medication without harm: WHO's Global Patient Safety Challenge. Lancet. 2017;39(10080):1680-1.

2. Ramacciato J, Motta RHL. Terapêutica Medicamentosa na Implantodontia. In: Perri PS, Pellizer EP. Fundamentos em Implantodontia - uma visão contemporânea. 2a ed. São Paulo: Quintessence; 2015.

3. Meiners MMMA, Bergsten-Mendes G. Prescrição de medicamentos para crianças hospitalizadas: como avaliar a qualidade? Rev Assoc Med Bras. 2001;47(4):332-7.

4. Armonia LP, Rocha RG. Como prescrever em Odontologia - marcas e genéricos. 9a ed. São Paulo: Santos; 2011.

5. Araújo LG, Biagini FC, Fernandes RL, Caputo IGC, Silva RHA. Conhecimento de acadêmicos de Odontologia sobre os aspectos clínicos, éticos e legais da prescrição medicamentosa. RFO UPF. 2012; 17(1):50-4.

6. Néri ED. Erros de prescrição de medicamentos em um hospital brasileiro. Rev Assoc Med Bras. 2011;57(3):306-14.

7. Ganashree P, Bhuvana K, Sarala N. Critical review of drug promotional literature using the World Health Organization guidelines. J Res Pharm Pract. 2016 Jul- Sep;5(3):162-5.

8. Cunha FL, Penna LAP, Soares HA, Varella MA. Anvisa eo Cirurgião- Dentista: do controle de qualidade de insumos às normas de prescrição de antimicrobianos. Rev Assoc Cir Paul Dent. 2011;65(4):252-7.

9. Castilho LS, Paixão HH, Perini E. Prescrição de medicamentos de uso sistêmico po cirurgiões-dentistas, clínicos gerais Rev Saúde Pública.1999;33(3):28794.

10. Carvalho VA, Borgatto AF Lopes LC. Knowledge level for prescription of nonsteroidal anti-inflammatory by dentists in São José dos Campos, São Paulo State, Brazil. Ciênc Saude Colet. 2010;15 Suppl 1:1773-82.

11. Jain A, Gupta D, Singh D, Garg Y, Saxena A, Chaudhary H, et al. Knowledge regarding prescription of drugs among dental students: a descriptive study. J Basic Clin Pharm. 2015;7(1):12-6.

12. Martín-Jiménez M, Martín-Biedma B, López-López J, Alonso-Ezpeleta O, Velasco-Ortega E, Jiménez-Sánchez MC, et al. Dental students' knowledge regarding the indications for antibiotics in the management of endodontic infections. Int Endod J. 2018;51(1):118-27.

13. Oberoi SS, Dhingra C, Sharma G, Sardana D. Antibiotics in dental practice: how justified are we. Int Dent J. 2015;65(1):4-10. 14. Pereira FM, Araújo MDO, Silva DRS, Santos AS, Lira EM, Nascimento LS. Antibiotic prophylaxis in Dentistry: a teaching experience using conceptual maps. Rev ABENO. 2018;18(1):155-160.

15. Moura CS, Naves JOS, Coelho EB, Lia EN. Assessment of quality of prescription by dental students. J Appl Oral Sci 2014;22(3):204-8.

16. Gelal A, Gumustekin M, Arici MA, Gidener S. Rational pharmacotherapy training for fourth-year medical students. Indian $\mathrm{J}$ Pharmacol. 2013;45(1):4-8.

17. Akram A, Zamzam R, Mohamad NB, Abdulah D, Meerah SM. An assessment of the prescribing skills of undergraduate dental students in Malaysia. J Dent Educ. 2012;76:1527-31.

18. Gadbury-Amyot CC, Singh AH, Overman PR. Teaching with technology: learning outcomes for a combined dental and dental hygiene online hybrid oral histology course. J Dent Educ. 2013;77(6):732-43. 
19. Gonzalez SM, Gadbury-Amyot CC. Using Twitter for teaching and learning in na Oral and Maxillofacial Radiology Course. J Dent Educ. 2016;80(2):149-55.

20. Barbosa ACS, Oliveira CVC, Gueiros MCN, Lima MC, Vasconcelos MMB, Caldas Júnior AF. Uso da tecnologia educacional web-based por profissionais da Odontologia brasileira. Rev ABENO. 2018;18(1):23-33.
21. Kamarudin G, Penm J, Chaar B, Moles R. Educational interventions to improve prescribing competency: a systematic review. BMJ Open. 2013;30;3(8):e003291.

\section{Correspondência para:}

Rogério Heládio Lopes Motta

e-mail: rogerio.motta@slmandic.edu.br

Rua José Rocha Junqueira, 13

13045-755 Campinas/SP 